\title{
Stereoregularity in Ionic Polymerization of Acenaphthylene
}

\author{
V. M. Story and G. Canty
}

(November 15, 1963)

\begin{abstract}
Four distinct polymers were isolated from the polymerizations of acenaphthylene initiated by boron trifluoride and $n$-butyllithium. A syndiotactic or isotactic conformation was assigned to these products on the basis of infrared and NMR evidence. The conformations and reaction details are discussed.
\end{abstract}

\section{Introduction}

A survey of the field of stereoregular polymers indicated that little has been done in deliberate control of chain conformation by means of steric hindrance of bulky groups [1].1,2 In this sense, acenaphthylene as a vinyl monomer was particularly interesting due to the extreme bulk and rigidity of the 1,8-perinaphthylene residue. Additional features were its strained five-membered ring [1a] and well defined conditions for polymerization.

The polymers of acenaphthylene have been extensively investigated. Dziewonski and his coworkers [2] were able to show ionic, thermal, and solid-state polymerization of acenaphthylene before the present-day tools for measuring high molecular weights were available. Their investigations started in 1912 and ended in 1924. Since then, other workers have examined these polymers. Jones [3] and Flowers and Miller [4], for example, have conducted extensive investigations. The only paper on the mechanism of this polymerization was by Imoto and Takemoto [5], using boron trifluoride etherate as an initiator. It should be emphasized that very little was done to measure molecular weights of these polymers [6] and no reference to possible stereoregularity was found.

A study of Fisher-Hirschfelder models of acenaphthylene polymers was made. The cis-isotactic polymer $\mathrm{I}$, where $\mathrm{A}$ is in

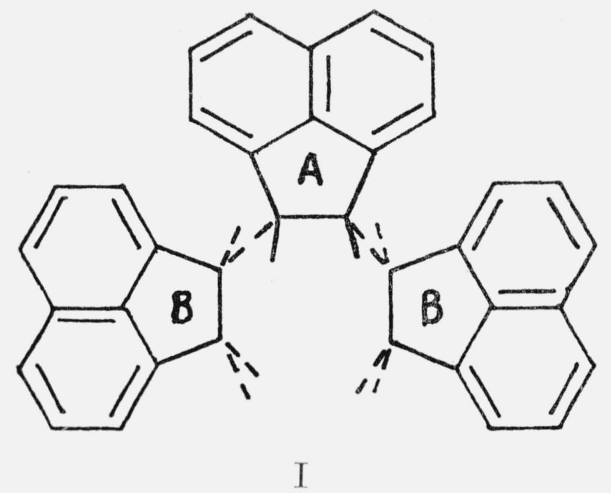

1 Figures in brackets indicate the literature references at the end of this paper. 2 Note added in proof: The paper by G. Natta, G. Dall'Asta, G. Mazzanti, and G. Metroni (Makromol. Chem. 69, 124, 1963) describes the polymerization of cyclobutene with Ziegler catalysts. The stereochemistry of the products are apparently identical with the case described here, Instead of cis or trans, the nomenclature threo or erythro, respectively, is used. the plane of the paper and the B's are in a parallel plane below, was self-terminating. Ring formation was favored. In fact, both a cyclic trimer and tetramer are known [2]. The cis-syndiotactic polymer II was impossible to construct beyond the trimer.

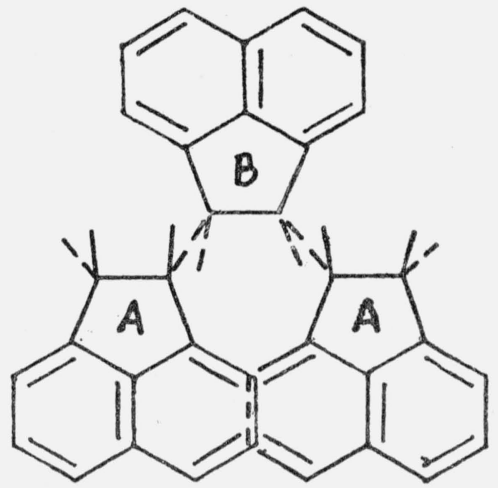

II

The same notation is used here as for I above. The A's were actually twisted out of plane due to steric hindrance. trans-Isotactic polyacenaphthylene, III, formed a helix. ${ }^{3}$

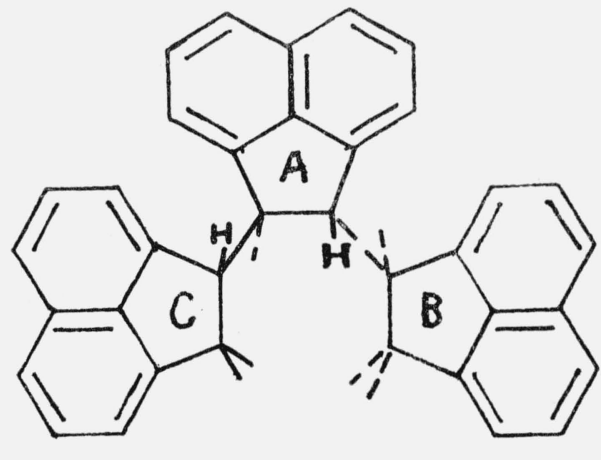

III

3 The limited rotation about the backbone carbon-carbon bonds due to steric hindrance of the 1,8-acenaphthylene residues suggests that change in the sense of the helix of III is not possible if a perultimate effect influences the course of of the helix of III is not possible if a penultimate effect influences the course of addition. Of course, a mixture of $d$ and $l$ chains would be produced, unless an
assymetric initiator were used. 
In this diagram, $\mathrm{C}$ is in a parallel plane above the paper, A is in it, and B below it. Similarly, the trans-syndiotactic polymer, IV, formed a "stairstepped" rigid rod. The same

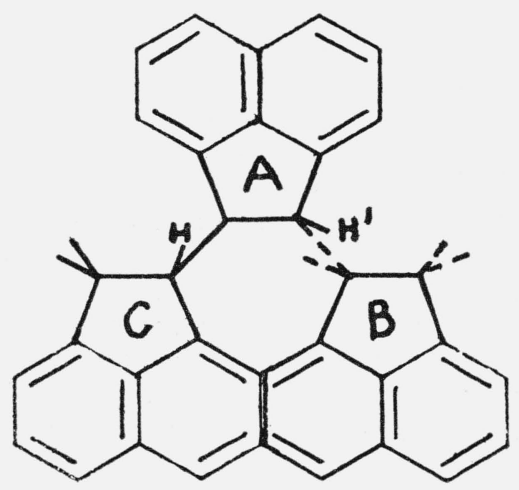

IV

notation is used here as in III. The tertiary aliphatic protons $(\mathrm{H})$ are on the inside of the helix in III and are 1,3-axial. In IV, these protons are isolated (to use the same nomenclature, they are 1,3equatorial) from each other. $\mathrm{H}^{\prime}$ is adjacent to the ortho aromatic protons of the next residue above (C) and to the $\pi$ cloud of the residue below (B).

The products of the polymerization of acenaphthylene with boron trifluoride and n-butyllithium initiators were investigated for conformations corresponding to III and IV above.

\section{Experimental Details}

\subsection{Materials}

Acenaphthylene (Aldrich Chemical Company) was sublimed twice in a vacuum apparatus. The melting point found was $92.5-93{ }^{\circ} \mathrm{C}$. The literature value is 92-93 ${ }^{\circ} \mathrm{C}$ [7]. Boron trifluoride (Matheson Company, Inc.) was transferred to a glass vacuumline and distilled twice, from a 2,2,4-trimethylpentane slush bath $\left(-107.4^{\circ} \mathrm{C}\right)$, to a trap cooled by liquid nitrogen. The vapor pressure of the redistilled boron trifluoride was found to be $458 \mathrm{~mm} \mathrm{Hg}$ at $-107.4{ }^{\circ} \mathrm{C}$. The literature value is $466 \mathrm{~mm} \mathrm{Hg}$ [8]. Chlorobenzene and benzene (Fisher Analyzed reagents) were used without further purification. An $n$-butyllithium preparation in $n$-heptane (Foote Mineral Company, Inc.) was used as received.

\subsection{Procedures}

\section{a. Polymerizations Initiated by Boron Trifluoride}

The acenaphthylene was weighed into a roundbottomed flask containing a Teflon-encased magnetic stirring-bar. Chlorobenzene was added and the flask was connected to a vacuum line. The flask contents were degassed by three freeze-pump-thaw cycles. A measured volume of boron triffuoride was transferred to the frozen solution. The flask contents were permitted to warm to the requiredreaction temperature with stirring. The color of the reaction mixture varied from deep green to black. On completion of the reaction, the flask was vented to the air. This did not cause much color change, and presumably did not destroy all of the complex. On pouring the flask contents into boiling methanol, all color disappeared (except that of residual monomer) and a dense, white precipitate coagulated from the reaction mixture. The precipitate was filtered off, washed with cold methanol, air-dried, and dissolved in benzene, and the solution was filtered through anhydrous sodium sulfate and freeze-dried. The filtrate from the original precipitation was vacuumevaporated. Any resulting residue was treated in the same way as for the original precipitate.

Co-initiators were added to the acenaphthylene solutions before connection to the vacuum line. In these cases, degassing was confined to one freezepump-thaw cycle.

A strong Tyndall effect was observed in all reaction mixtures. However, no solid separated out during or after the reactions. When sufficient water was used as a co-initiator, it was observed as ice droplets in solution or film on the sides of the flask.

\section{b. Polymerization Initiated by $n$-Butyllithium}

The reaction was conducted as above, except that the $n$-butyllithium solution was added through a rubber disk with a hypodermic syringe and needle. Again, the complex formed was dark green to black in color. Admission of air immediately destroyed all traces of color. After the solution had been vented to the air, it was extracted twice with $0.1 \mathrm{~N}$ $\mathrm{HCl}$ and washed three times with water. The organic layer was then treated as in (a) above, by precipitation in boiling methanol, etc.

\subsection{Physical Properties}

Intrinsic viscosities were determined in the standard manner at $35^{\circ} \mathrm{C}$ in benzene. Infrared spectra were determined on a Perkin-Elmer 221 with a grating interchange. NMR spectra were obtained, for all polymers isolated, on a Varian A-60 instrument. Carbon tetrachloride was used as the solvent [11] and tetramethylsilane as the external standard. Ultraviolet spectra were made on a Cary Model 14 in benzene at $25{ }^{\circ} \mathrm{C}$. X-ray photographs of powder specimens were taken with a Debye-Scherrer camera, using copper $K \alpha$ radiation.

\section{Results and Discussion}

The results of the polymerizations of acenaphthylene, initiated by boron trifluoride and $n$-butyllithium with and without cocatalysts, are listed in table 1. 
TABLE 1. Ionic polymerization of acenaphthylene

\begin{tabular}{|c|c|c|c|c|c|c|}
\hline \multirow{2}{*}{$\begin{array}{l}\text { Reaction } \\
\text { number }\end{array}$} & \multirow{2}{*}{$\begin{array}{c}\mathrm{C}_{12} \mathrm{H}_{8} \\
{[M]^{2,3}}\end{array}$} & \multirow{2}{*}{$\begin{array}{l}\text { Initiator, } \\
{[I] \times 10^{4}}\end{array}$} & \multicolumn{2}{|c|}{ Co-initiator } & \multirow{2}{*}{$\begin{array}{l}\text { Reaction } \\
\text { time }\end{array}$} & \multirow{2}{*}{ Yield } \\
\hline & & & type & {$\left[\stackrel{\text { cone. }}{M]} \times 10^{4}\right.$} & & \\
\hline
\end{tabular}

I. Initiation by boron trifluoride

\begin{tabular}{|c|c|c|c|c|c|c|}
\hline 1 & 2.35 & const. ${ }^{4}$ & $\mathrm{H}_{2} \mathrm{O}_{5}$ & & $45 \mathrm{~min}$ & $92.4^{6}$ \\
\hline 2 & 2.35 & const. ${ }^{4}$ & $\mathrm{H}_{2} \mathrm{O}^{5}$ & & 4 days & $82.4^{6}$ \\
\hline 3 & 2.35 & const. ${ }^{4}$ & $\mathrm{H}_{2} \mathrm{O}_{5}^{5}$ & & 6 days & $89.0^{6}$ \\
\hline 4 & 2.35 & const. ${ }^{4}$ & $\mathrm{H}_{2} \mathrm{O}^{5}$ & & 8 days & $90.1^{6}$ \\
\hline 5 & 0.211 & 48.1 & none 7 & & $30 \mathrm{~min}$ & 84.2 \\
\hline 6 & .211 & 45. 0 & none & & $30 \mathrm{~min}$ & 87.0 \\
\hline 7 & .211 & 35.1 & none & & $30 \mathrm{~min}$ & 86.8 \\
\hline 8 & .211 & 11.3 & none & & $30 \mathrm{~min}$ & 74.3 \\
\hline 9 & .211 & 6.67 & none & & $30 \mathrm{~min}$ & 82.3 \\
\hline 10 & .211 & 32.7 & $\mathrm{CH}_{3} \mathrm{OH} 7$ & 2.4 & $30 \mathrm{~min}$ & $30.2^{8}$ \\
\hline 11 & 0.201 & 8.85 & $\mathrm{CH}_{3} \mathrm{OH}^{7}$ & 61.8 & $30 \mathrm{~min}$ & 0.0 \\
\hline 12 & .201 & 29.1 & $\mathrm{H}_{2} \mathrm{O}^{7}$ & 55.6 & $22 \mathrm{~min}$ & 69.1 \\
\hline 13 & 0.343 & 17.4 & $\mathrm{H}_{2} \mathrm{O}^{7}$ & 139 & $30 \mathrm{~min}$ & 82.4 \\
\hline 14 & .358 & 13. 9 & $\mathrm{H}_{2} \mathrm{O}^{7}$ & 556 & $30 \mathrm{~min}$ & 49. 6 \\
\hline 15 & .516 & 12.2 & $\mathrm{H}_{2} \mathrm{O}_{9}^{9}$ & & $30 \mathrm{~min}$ & 87.6 \\
\hline
\end{tabular}

II. Initiation by $n$-butyllithium 10

\begin{tabular}{|c|c|c|c|c|c|}
\hline $\begin{array}{l}16 \\
17\end{array}$ & $\begin{array}{r}0.075 \\
.028\end{array}$ & $\begin{array}{l}6.0 \\
30^{-}\end{array}$ & $(7)$ & $\begin{array}{l}24 \mathrm{hr} \\
24 \mathrm{hr}\end{array}$ & $\begin{array}{l}7.0 \\
0.7\end{array}$ \\
\hline
\end{tabular}

$1[I]$ indicates the concentration (moles per liter) of ionic initiator if it all dissolved in the monomer solution.

2 The solvent for these reactions was chlorobenzene. Reactions 1-4 were done at $-5^{\circ} \mathrm{C}$ and the remainder at $-23^{\circ} \mathrm{C}$

$3[M]$ indicates the concentration of acenaphthylene in moles per liter.

A slow flow of $\mathrm{BF}_{3}$ was supplied to the reaction mixture.

Traces of water were present.

6 These reaction products were precipitated from cold methanol.

${ }^{7}$ See experimental section for details.

8 The product soluble in boiling methanol was $30.2 \%$, the insoluble, $2.0 \%$.

9 Moist air was admitted to the reaction flask during the run.

10 The solvent was benezene. The reaction temperature was $21{ }^{\circ} \mathrm{C}$. The calculated molarity of $n$-butyllithium in the final solution is reported.

The first four reactions in table 1 duplicate the procedure of Flowers and Miller [4]. It was found that the yields from precipitation of the reaction mixture in cold methanol were not reproducible. The polymeric product was observed to redissolve on standing in the methanol-chlorobenzene mixture. An exhaustive extraction of a portion of polymer precipitated in the above manner showed an 11 percent weight loss. Since the methanol-soluble material showed the same spectra as the parent polymer in the ultraviolet region, it was concluded that oligomers were being extracted. To circumvent the coprecipitation of the oligomers, hot (boiling) methanol was used as the precipitating medium. The precipitated polymer isolated by this method will be designated "insoluble" and the product isolated from the supernatant hot methanolchlorobenzene solution by cooling and vacuum evaporation will be designated "soluble" in the following discussion.

The residue left after isolating both the "insoluble" and "soluble" polymers was determined to be 90 to 95 percent monomer in all cases. The "insoluble" polymer isolated in the above series was designated type $\mathrm{G}$.

Reactions 5 through 9, table 1, were performed using anhydrous boron trifluoride as initiator. The average total recoverv, calculated on the basis of monomer weight, was 97.6 percent. The "soluble" polymer isolated in reaction 7 proved to be similar to the "insoluble" one, but of lower molecular weight.
When water or moist air was used as a cocatalyst (reactions 12 through 15 , table 1), the pooled "soluble" polymer for reactions 13, 14, and 15 showed properties different from those of the "insoluble" polymer obtained in the same reactions, as described below in terms of their respective physical properties. It was designated type $\mathrm{K}$.

The most striking change in products was observed when a small amount of methanol was added as co-initiator. In this case (reaction 10, table 1), the "soluble" polymer was the predominant component and proved to be a different macromolecular species, designated type M. The small amount of "insoluble" polymer recovered in this reaction was type G. Increase of the concentration of methanol co-initiator completely inhibited polymer formation, as the data for reaction 11 in table 1 indicate.

The polymerizations of acenaphthylene initiated by $n$-butyllithium gave low yields for the conditions chosen, as shown in table 1 , reactions 16 and 17 . The separation procedure given above for the polymerizations initiated by boron trifluoride did not give definitive results, because of low yields. The product isolated was different from all others described above and was designated type $\mathrm{N}$.

The physical properties of the polymer types described above are summarized in table 2 .

TABLE 2. Polymeric products of polymerization of acenaphthylene

\begin{tabular}{|c|c|c|c|c|}
\hline $\begin{array}{l}\text { Polymer } \\
\text { type } 1\end{array}$ & $\begin{array}{l}\text { Reaction } \\
\text { (table 1) }\end{array}$ & $\begin{array}{c}\text { Solu- } \\
\text { bility }{ }^{2}\end{array}$ & $3[\eta]]_{\mathrm{C}_{6} \mathrm{H}_{6}}^{35^{\circ}}$ & $\epsilon_{A^{4}}$ \\
\hline G & $\begin{array}{l}1-4 \\
5-9 \\
7 \\
10\end{array}$ & $\begin{array}{r}0 \\
0 \\
+ \\
0\end{array}$ & $\begin{array}{c}0.039 \pm 0.004 \\
.034 \pm 0.001 \\
.025^{5} \\
(6)\end{array}$ & $\begin{array}{l}6860 \\
6870 \\
6570\end{array}$ \\
\hline $\begin{array}{l}\mathrm{K} \\
\mathrm{M} \\
\mathrm{N}\end{array}$ & $\begin{array}{l}12-15 \\
12-15 \\
10 \\
16,17\end{array}$ & $\begin{array}{l}0 \\
0 \\
+7 \\
+\quad \\
(9)\end{array}$ & $\begin{array}{l}.047 \pm 0.002 \\
.029 \\
.028 \\
.036,0.1099\end{array}$ & $\begin{array}{l}6890 \\
6500 \\
5900 \\
6080\end{array}$ \\
\hline
\end{tabular}

1 The polymer types are as designated in the text.

2 The solubility refers to the boiling methanol chlorobenzene mixture from which the polymer is precipitated, as described in the experimental section. Reactions 1-4 were precipitated in cold methanol.

3 Two-point viscometric determinations were made. The $[\eta]$ values were cor${ }^{3}$ Two-point viscometric determinations were made. The [ $\left.\eta\right]$ values were cor-
rected for the presence of $11 \%$ oligomer for reactions $1-4$ and all "soluble" prodrected for the presence of $11 \%$ oligomer for reactions $1-4$ and all "soluble" prod-
ucts (see text). The mean and the average deviation from the mean is reported. ucts (see text). The mean and the average deviation from the mean is reported.
4 The extinction coefficient was calculated per acenaphthylene residue. The determinations were done in benzene at $2960 \AA$ and at $25^{\circ} \mathrm{C}$. The average error was $2 \%$.

5 The physical properties were determined on one sample, reaction 7 , table 1 . 6 Product was identified by its infrared spectrum. See text for details.

7 The properties of a pooled sample were determined.

8 The yield was too low to permit determination of the solubility.

9 The intrinsic viscosities of the two products were widely different, due to 9 The intrinsic viscosities of the two prod
varying experimental conditions (table 1 ).

\subsection{Physical Properties}

\section{a. Viscosities and Molecular Weights}

Several molecular-weight determinations were made, to index the viscosity determinations given in table 2. An osmotic-pressure determination of one of the polymerization products $\left([n]_{\mathrm{C}_{6} \mathrm{H}_{6}}^{33 \circ}=0.036\right.$, reaction 1 , table 1 ) initiated by boron trifluoride gave the value 7,070 for a number-average molecular weight. The large effect of molecules of very low molecular weight on this type of measurement led us to make the same determination with a vaporpressure osmometer. The value 2,670 was obtained 
by this means. This is reasonable in view of the low molecular weights and the absence of extensive purification. Taking 3,000 as an approximate number-average molecular weight, the mean degree of polymerization was about 20 .

Two groups of workers have reported on the number-average molecular weight of polymers of acenaphthylene initiated by boron trifluoride. Flowers and Miller [4] stated that the moleuclar weight by osmometry was about 150,000. Imoto and Takemoto [6] reported an intrinsic viscosity of 0.040 $\left(30^{\circ} \mathrm{C}\right.$, in benzene) and a number-average molecular weight of 125,000 using a collodion membrane. Both the above papers mention "repeated reprecipitation in methanol" from benzene solutions. However, the intrinsic viscosity value reported in the second paper indicated that extensive purification was not obtained, compared to the methods used in this study. We were not able to resolve these large discrepancies.

\section{b. Infrared Spectra}

The saturated, tertiary carbon atoms of the backbone of polyacenaphthylene would be expected to show different $\mathrm{C}-\mathrm{H}$ stretch frequencies for the trans-syndiotactic and trans-isotactic polymers. Figures 1 and 2 show the $\mathrm{C}-\mathrm{H}$ stretch spectra corresponding to the four polymer types isolated in this investigation. Polymer type $\mathrm{G}$, table 2 , corresponds to spectrum $A$, figure 1 ; type $K$ to spectrum $B$; type $\mathrm{M}$ to $\mathrm{C}$; and type $\mathrm{N}$ to $\mathrm{D}$ and $\mathrm{E}$ in figure 2.

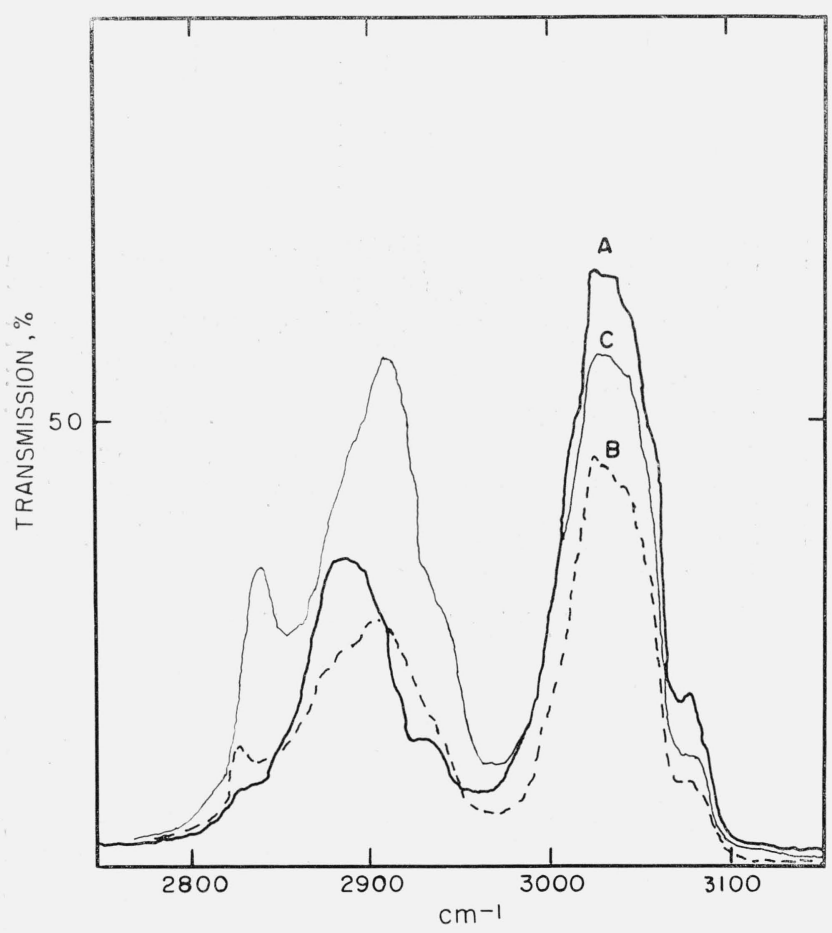

Figure 1. Type $G$ polymer, spectrum A; type $K, B$; and type $M, C$.

Ordinates, $\mathrm{cm}^{-1}$; abcissae in arbitrary percent transmission.

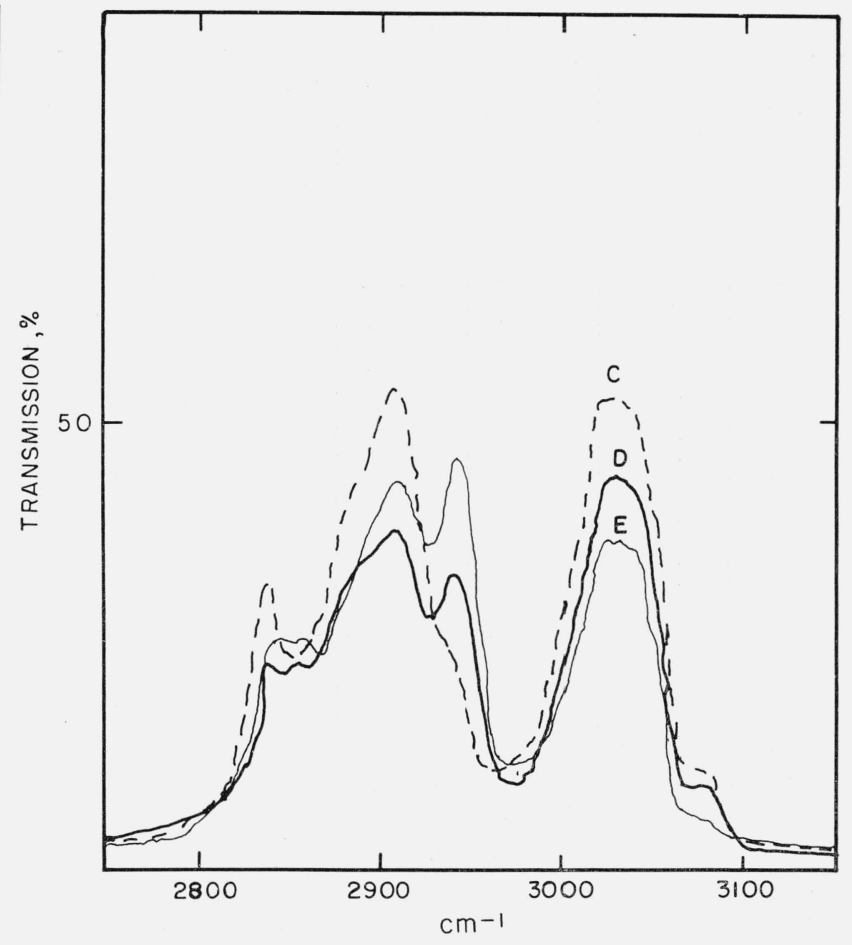

Figure 2. Type $M$ polymer, spectrum C; type $N$, spectra $\mathrm{D}$ and $\mathrm{E}$

$\mathrm{D}$ is the spectrum of the product isolated from reaction 16, table 1, and $\mathrm{E}$ of that from reaction 17. Ordinates, $\mathrm{cm}^{-1}$; abcissae in arbitrary percent transmission.

The frequencies of the aliphatic $\mathrm{C}-\mathrm{H}$ stretch bands of these spectra are listed in table 3, together with those of the cis- and trans-declains and acenaphthene. Although the frequencies are accurate to $\pm 5 \mathrm{~cm}^{-1}$ in this study, comparison with other spectra from the literature are probably accurate to only $\pm 10 \mathrm{~cm}^{-1}$ because of resolution differences and changes of state.

The assignment of the aliphatic $\mathrm{C}-\mathrm{H}$ stretch frequency of type $\mathrm{G}$ polyacenaphthylene is straightforward and unambiguous, since it is almost exactly the $2890 \pm 10 \mathrm{~cm}^{-1}$ for tertiary $\mathrm{C}-\mathrm{H}$ established by Fox and Martin [9]. This frequency assignment corresponds to the syndiotactic trans polymer (IV), as an isolated tertiary aliphatic $\mathrm{C}-\mathrm{H}$ bond is unique to this structure.

Spectrum B, figure 1, contains frequencies common to both $\mathrm{A}$ and $\mathrm{C}$. We will discuss spectrum $\mathrm{C}$ first, to clarify its assignments, before considering $\mathrm{B}$.

A Fisher-Hirschfelder model for the trans-isotactic polymer, III, required 1,3-axial proton interactions similar to those of the axial bonds of cyclohexane. The closest analogs found were cis- and transdecalin. As shown in table 3 , the asymmetric $\mathrm{C}-\mathrm{H}$ stretch frequency of spectrum $\mathrm{C}$ (type $\mathrm{M}$ polymer) is at $2908 \mathrm{~cm}^{-1}$, which is $16 \mathrm{~cm}^{-1}$ below that of cis- and trans-decalin at $2924 \mathrm{~cm}^{-1}$. By invoking a limited degree of chain flexibility for polymer configuration III, the decrease in frequency can be attributed to lower " 1,3 " proton-interaction energy as compared to that of the model compounds. 
TABLE 3. Aliphatic $\mathrm{C}-\mathrm{H}$ infrared frequencies ${ }^{1}$

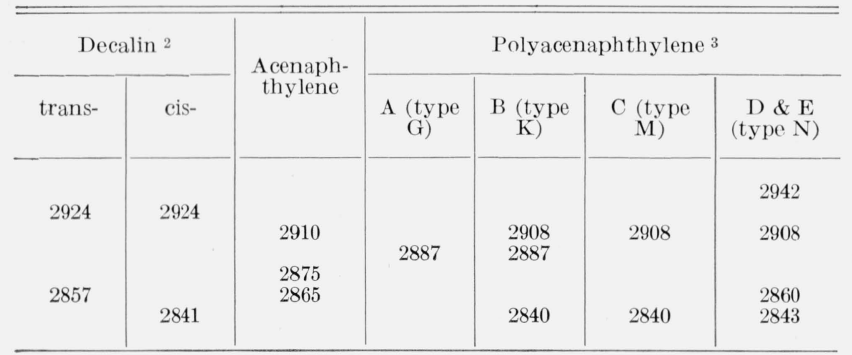

1 The frequencies are given in $\mathrm{cm}^{-1}$; the solvent was carbon tetrachloride, unless otherwise stated.

2 From American Petroleum Institute, Infrared Spectra 1086 and 1087 respectively. These were spectra taken on pure liquid samples.

3 The letter designations correspond to the spectra in figures 1 and 2 . The type designations are given in the text.

The symmetric $\mathrm{C}-\mathrm{H}$ stretch frequency at 2840 $\mathrm{cm}^{-1}$ for spectrum $\mathrm{C}$ corresponds exactly with that of cis-decalin. As has been shown by nuclear magnetic resonance spectra [10], cis-decalin has one averaged proton-proton interaction, since the ring system is flexible. This fact suggests that the aliphatic protons in polymer $\mathrm{M}$ are predominantly 1,3-axial and do not interact with aromatic protons.

With the above assignments in mind, spectrum B can be rationalized as indicating the presence of either a mixture of types $\mathrm{G}$ and $\mathrm{M}$, or a block copolymer. Spectra D and E, figure 2, corresponding to type $\mathrm{N}$ polymer, are clearly related to spectrum $\mathrm{C}$, but have two more peaks, at 2860 and $2942 \mathrm{~cm}^{-1}$. The first of these can be assigned to the $\mathrm{C}-\mathrm{H}$ stretch frequency of the $\mathrm{CH}_{2}$ group, as shown for trans-decalin, table 3 , and the second to a combination of $\mathrm{CH}_{2}$ and $\mathrm{CH}_{3}$ frequencies. This reasoning is based on the assumption that $n$-butyl groups, derived from the $n$-bucyllithium initator, were appended to one end of the polymer chain.

\section{c. Nuclear Magnetic Resonance (NMR)}

Acenaphthene was investigated as a reference compound under the same conditions as for the polymers. This compound has nine peaks in the aromatic proton range, from 7.07 to 7.54 parts per million of the applied magnetic field (ppm) $[12,13]$ and a sharp peak at $3.23 \mathrm{ppm}$ corresponding to the secondary aliphatic protons. The latter value is higher than that for aliphatic protons on alkyl groups; for instance, a cyclohexane NMR spectrum gives $1.43 \mathrm{ppm}$ [14]. Acenaphthene is known to have a strained ring [1a], and this fact possibly accounts for a higher screening-constant, because of reduction of the bond angle between the geminal protons.

The aromatic-proton peaks characteristic of acenaphthenewere smeared into a broad peak in the same location for all of the polymer spectra. Only type G and type $M$ polymers showed the sharp peaks characteristic of the aliphatic protons. The field shift for type $\mathrm{G}$ was $7.25 \mathrm{ppm}$, and for type $\mathrm{M}$, $1.20 \mathrm{ppm}$.

The model studies and infrared assignments above required that the III structure have its tertiary aliphatic protons 1,3-axial to each other and on the inside of a helix. Close examination of such structures led to the conclusion that the nearest neighbor interactions were small, due to the flexibility of the backbone. The value found for $\mathrm{M}, 1.20 \mathrm{ppm}$, is in complete agreement with this reasoning, and comparison with the NMR spectra of cyclohexane (loc. cit.) supported this argument.

The structure predicted for G, IV above, showed close proximity of the tertiary protons to the $\pi$ electron "cloud" and to aromatic protons of adjacent aromatic residues. The chemical shift is expected to be high due to high screening of aromatic rings. The value found for $G, 7.25 \mathrm{ppm}$, is in accord with structure IV.

These NMR spectra complemented the infrared assignments and indicated that the tertiary-proton environments are those predicted from the model studies of polymers $\mathrm{G}$ and $\mathrm{M}$. Type $\mathrm{K}$ polymer remained indeterminate in structure, since no further information was acquired as to whether it was a mixture or a copolymer. The NMR spectrum for type $\mathrm{N}$ was not resolved, due, no doubt, to its higher molecular weight. (High-temperature equipment was not available to increase the resolution.) However, the infrared evidence indicated that $\mathrm{N}$ is a derivative of III.

\section{d. Ultraviolet Spectra}

It would be expected that trans-isotactic polyacenaphthylene would be less accessible to solvent interaction, due to its helical conformation (III), than the trans-syndiotactic polymer (IV) and thus show a lower extinction coefficient. The results shown in table 2 support this contention. These data are considered here to be supplementary supporting evidence for the proposed configurations.

\section{e. X-ray spacings}

Powder specimens of polyacenaphthylene designated type $G, M$, and $N$ gave the Bragg spacings shown in table 4 . The powder photographs were

TABLE 4. Bragg spacings for polyacenaphthylene ${ }^{1}$

\begin{tabular}{c|c|c|c}
\hline \hline Type G & Type M & Type N & $\begin{array}{c}\text { Relative } \\
\text { intensity 2 }\end{array}$ \\
\cline { 1 - 1 } & & & \\
2.07 & 2.07 & 2.06 & $\mathrm{vw}$ \\
2.81 & 2.76 & 2.75 & $\mathrm{w}$ \\
3.95 & 3.91 & 3.95 & $\mathrm{~S}$ \\
5.47 & 5.47 & 5.27 & $\mathrm{~m}$ \\
10.3 & 10.8 & 10.4 & $\mathrm{vs}$ \\
& &
\end{tabular}

The spacings are given in angstrom units, and the type designations are given in the text.

$2 \mathrm{vw}$ indicates very weak, w weak, $m$ medium, s strong, and, vs very strong relative intensities.

not sharp. The larger spacings useful for determining the chain conformation were not resolved. There were several spacings which were significantly different among the three polymer types. The 2.81 $\AA$ spacing for type $\mathrm{G}$, and the $5.27 \AA$ spacing for type $\mathrm{N}$ were clearly different. The $10.8 \AA$ spacing for type $M$ was not significantly different from the 
10.3 and $10.4 \AA$ spacings for types $\mathrm{G}$ and $\mathrm{N}$, respectively. Thus, although differences were apparent, no definitive analysis could be made of these data to determine the crystal structures of the polymer types.

\subsection{Mechanism}

It is useful to consider the chemical nature of initiator and co-initiator complexes before inquiring into the polymerization process. Greenwood and Martin have shown that stable complexes are formed when boron trifluoride reacts with water or methanol [15]. They have also shown that the compounds hydroxyfluoroboric acid $\left(\mathrm{H}^{+} \mathrm{BF}_{3} \mathrm{OH}^{-}\right)$, dihydroxyfluoroboric acid $\left(\mathrm{H}_{3} \mathrm{O}^{+} \mathrm{BF}_{3} \mathrm{OH}^{-}\right)$, methoxyfluoroboric acid $\left(\mathrm{H}^{+} \mathrm{BF}_{3} \mathrm{OCH}_{3}^{-}\right)$, and dimethoxyfluoroboric acid $\left(\mathrm{H}_{2} \mathrm{OCH}_{3}{ }^{+} \mathrm{BF}_{3} \mathrm{OCH}_{3}^{-}\right)$have definite and large conductivities in the melt [16]. These data are reported in table 5 in terms of percent ionization in the melt, together with the melting points of the fluoroboric acid compounds measured by the same authors. As solids, these compounds are reported to be partially ionized if crystallized rapidly from solution [17]. Other evidence [18] favors the ionic structures indicated in the empirical formulas. The relative amounts of ionization of the methoxyfluoroboric acids are supported by the calculations of Bender [19] from the equation of Edwards [20]. Bender's calculations indicate that methanol is a stronger nucleophile than water. Thus, the methoxyfluoroboric acid compounds would be expected to show less ionization than the comparable hydroxyfluoroboric acids, in agreement with the results in table 5 .

Table 5. Properties of fluoroboric acid complexes ${ }^{1}$

\begin{tabular}{c|c|c}
\hline \hline Acid complex & MP & Ionization 2 \\
& & \\
$\mathrm{H}^{+} \mathrm{BF}_{3} \mathrm{OH}^{-}$ & ${ }^{\circ} \mathrm{C}$ & $\%$ \\
$\mathrm{H}_{3} \mathrm{O}^{+} \mathrm{BF}_{3} \mathrm{OH}^{-}$ & $5.9-6.0$ & 10 \\
$\mathrm{H}^{+} \mathrm{BF}_{3} \mathrm{OCH}_{3}^{-}$ & 6.2 & 20 \\
$\mathrm{H}_{2} \mathrm{OCH}_{3}+\mathrm{BF}_{3} \mathrm{OCH}_{3}-$ & -18.6 & 2 \\
\hline
\end{tabular}

1 The references are given in the text.

2 The ionization was measured in the melt at $20^{\circ} \mathrm{C}$.

On the basis of infrared spectra, the greater ionization of the dihydroxyfluoroboric and dimethoxyfluoroboric acids as compared to the corresponding mono-acids (table 5) is attributed by Babushkin et al., to dimer formation, as contrasted to polymer formation for the 1:1 compounds [21]. Diehl [22] reports NMR evidence in accord with this conclusion.

Comparison of the melting points for the fluoroboric acid complexes given in table 5 with the experimental conditions for acenaphthylene polymerization given in table 1 suggest that all but one of the reactions initiated by boron trifluoride were conducted under heterogeneous conditions. As Clark has pointed out [25], traces of water are present even when extreme care is taken to produce 'anhydrous' boron trifluoride for vinyl polymerizations. The observed Tyndall effect must be due to the initiator complex present as a finely divided solid since the polymer is soluble in chlorobenzene. Thus, the fluoroboric acid compounds are solid under the given reaction conditions, as was excess water, but not methanol. In reaction 11 , the initiator complex would be dissolved in excess methanol, in contrast to 10 where heterogeneous initiation is expected.

Several attempts were made to determine the rates of polymerization of acenaphthylene under the conditions of experiment 6 , table 1 . Reaction times as short as $20 \mathrm{sec}$ gave the same yields. It was not feasible to extend these experiments to shorter reaction times.

The deliberate addition of water or methanol had a variable effect on the yield (reactions 10 through 15). With the exception of reaction 13, the excess of co-initiator over the initiator (on a molar basis) depressed the yield as well as changed the nature of the product. These facts suggest that the addition of co-initiator inhibits the formation of both polymers, but that the inhibition is greater for the isotactic product. The exact cause of the increase in yield of reaction 13 compared to 12 and 14 is not known.

Reactions 1 through 4 are comparable to reactions 6 through 9 in vield when corrected for oligomer concentration. These reactions were probably initiated by hydroxyfluoroboric acid while reactions 12 through 15 were initiated predominantly by dihydroxyfluoroboric acid. The degree of polymerization of reactions 12 through 15 was higher (table 2 ) with respect to the insoluble product (type G).

The above discussion is certainly oversimplified, since both hydroxyfluoroboric acids are present at all times, but in different proportions [18]. However, we have argued in terms of the predominant species for the sake of clarity.

Before proceeding to the case of acenaphthylene polymerization by initiation with methoxyfluoroboric acid, it is profitable to review the details of acenaphthylene polymerization initiated by hydroxyfluoroboric acid. Consideration of the pertinent data in table 1 show that temperature (footnote 3 , table 1), monomer concentration, and reaction time have no discernible effect on the yield under the given reaction-conditions. The degree of polymerization of the products increases when the water concentration increases. The extinction coefficients for the polymers given in table 2 indicate that small amounts of the trans-isotactic polymer are present in the "soluble" product. Whether or not the isotactic polymer is or is not present as part of a block copolymer remains undecided. However, a block copolymer is unlikely at these low degrees of polymerization. The primary conclusion from the structure assignments and discussion above is that solid hydroxyfluoroboric acids give, predominantly, trans-syndiotactic polyacenaphthylene. It is probable, but not so well established, that the stronger dihydroxyfluoroboric acid decreases the yield, but increases the degree of polymerization.

With the above summary in mind, the heterogeneous initiation of acenaphthene polymerization by methoxyfluoroboric acid (reaction 10, table 1) has only one difference from initiations by the hydroxy- 
fluoroboric acids. As shown in table 5, methoxyfluoroboric acid is a distinctly weaker acid or, alternatively, a stronger nucleophile. The predominant polymer found is trans-isotactic polyacenaphthylene, with traces of the trans-syndiotactic polymer, in contrast to the polymerizations of acenaphthylene by hydroxyfluoroboric acids. It follows that the methoxyfluoroboric acid anion stabilizes the carbonium ion formed after donating its proton to acenaphthylene. Furthermore, the propagating polymer carbonium ion must be stabilized. The increase in nucleophilicity of the methoxyfluoroboric acid over the hydroxyfluoroboric acids is enough to favor formation of the trans-isotactic polymer carbonium ion over the corresponding trans-syndiotactic carbonium ion. The high steric hindrance of the trans-isotactic carbonium ion indicates that a "sufficiently stable" complex must last longer than the comparable syndio carbonium ion, since three of the six Cartesian axes are accessible to monomer attack, compared to four in the transsyndio carbonium ion.

Turning now to acenaphthylene polymerization by $n$-butyllithium, we find a clear precedent set for us. Worsfold and Bywater [23] have shown that previously reported cases of stereoregular polymerization of styrene [24] by $n$-butyllithium in aromatic solvents were dependent on the presence of traces of water. The experiments reported here did not rigorously exclude water in the acenaphthene polymerization with $n$-butyllithium initiation. The product obtained was trans-isotactic polyacenaphthylene with $n$-butyl end-groups (loc. cit.).

Although Worsfold and Bywater [23] did not believe that their experiments decided the question of "whether a colloidal surface is necessary," their reactions were run at $-30{ }^{\circ} \mathrm{C}$ where any added water is certainly frozen to ice. The generation of lithium $n$-butoxide without complete destruction of the initiator probably creates a situation analogous to that discussed above, where mixtures of solid compounds act as stereoregular initiators. The same reasoning applies in detail to reactions 15 and 16 in table 1.

The discussion above leads to the conclusion that all of the effective initiators used were heterogeneous. The nucleophilicity of the fluoroboric acid complex determined the stability of the initiating and propagating carbonium ion and, hence, the predominant stereoregular product. Traces of ice reacting with $n$-butyllithium were probably instrumental in inducing heterogeneous initiation of acenaphthylene polymerization.

\section{Summary}

The four polymers isolated from acenaphthylene polymerizations initiated by boron trifluoride and $n$-butyllithium were shown to correspond to syndiotactic or isotactic structures on the basis of infrared and NMR spectra. The syndiotactic polymer had a "rod" conformation, and the isotactic, a helix.
We thank J. H. O'Mara for determining the osmotic pressure and W. T. Meyer for determining some of the viscosities. A. Diorio kindly made the powder x-ray photographs. We appreciate helpful discussions with Dr. J. P. Kennedy of Esso Research and Engineering Co., Ine.

\section{References}

[1] M. Farina and G. Byessan, Makromol. Chem. 61, 79 (1963).

[1a] H. W. Ehrlich, Acta Cryst. 10, 699 (1957).

[2] K. Dziewonski and Z. Lylso, Ber. 47, 1769 (1914); K. Dziewonski and T. Stolyluwo, ibid. 57, 1531 (1924).

[3] J. I. Jones, J. Appl. Chem. 1, 568 (1951).

[4] R. G. Flowers and H. F. Miller, J. Am. Chem. Soc. 69, 1388 (1947).

[5] M. Imoto and K. Takemoto, J. Polymer Sci. 79, 271 (1955).

[6] Reference 3, 4, and M. Imoto and I. Somatsu, Bull Chem. Soc. Japan 34, 26 (1961).

[7] A. Behr and W. A. van Dorp, Ber. 6, 60, 753 (1873); Ann. 17\%, 263 (1874); A. Behr and M. Blumenthal, Ber. 7, 1092 (1874).

[8] O. Ruff, A. Braida, O. Bretschneider, W. Menzel, and H. Plaut, Z. Anorg. Allgem. Chem. 206, 59 (1932).

[9] J. J. Fox and A. E. Martin, Proc. Roy. Soc. (London), A175, 208 (1940).

[10] J. A. Pople, W. G. Schneider, and H. J. Bernstein, High resolution nuclear magnetic resonance spectroscopy, pp. 399 (McGraw-Hill Book Co., New York, N. Y., 1959).

[11] For a discussion of errors due to this type of standardization, see reference 12 , pp. 78,87 , and 98 .

[12] $\delta(\mathrm{ppm})=\frac{H_{\mathrm{TMS}}-\mathrm{H}}{H_{\mathrm{TMS}}}=\frac{V-V_{\mathrm{TMS}}}{60}=\frac{\mu}{60}$, the chemical shift, where $H$ refers to the magnetic-field shift, $V$ is the field shift in cycles per second, and the subscript TMS refers to tetramethylsilane.

[13] Benzene gives $\delta=7.37$. Spectrum N-53, Catalogue of NMR spectra (Varian Associates, Inc., 1962, Palo Alto, Calif.).

[14] Reference 15, Spectrum N-122.

[15] N. N. Greenwood and R. L. Martin, J. Chem. Soc. 1951, 1915.

[16] N. N. Greenwood and R. L. Martin, J. Chem. Soc. 1953, $1427,757$.

[17] P. T. Ford and R. E. Richards, J. Chem. Soc. 1956, 3870 .

[18] J. A. Nieuland, R. R. Vogt, and W. H. Foohey, J. Am. Chem. Soc. 52, 1018 (1930); L. J. Klinkenberg and J. A. A. Ketelaar, Rec. trav. chim. 54, 959 (1935); J. L. Hoard, S. Geller, and T. B. Owen, Acta Cryst. 4, 405 (1951); J. F. MeKenna and F. J. Sowa, J. Am. Chem. Soc 59, 470 (1937); N. F. Toussant and G. F. Heunion, ibid. 62, 1145 (1940); C. E. Welsh and G. F. Hennion, ibid. 63, $2603(1940)$

[19] M. L. Bender, Chem. Revs. 60, 62 (1960).

[20] J. O. Edwards, J. Am. Chem. Soc. 78, 1819 (1956).

[21] A. A. Babushkin, L. A. Gribov, N. G. Guseva, and V. M. Emel'yanova, Opt. i Spektroskopiya, 5, 256 (1958).

[22] P. Diehl, Helv. Phys. Acta 31, 385,43 (1958).

[23] D. J. Worsfold and S. Bywater, Makromol. Chem. 65, 245 (1963).

[24] D. Braun W. Betz, and W. Kern, Makromol. Chem. 42, 89 (1960); R. J. Kern, Nature (London) 187, 410 (1960).

[25] D. Clarke in Cationic polymerization and related complexes, p. 90, P. H. Plesch, Ed. (Heffner, Cambridge, England, 1953).

(Paper 68A2-265) 